\title{
Second-order cone programming formulations for a class of problems in structural optimization
}

\author{
Athanasios Makrodimopoulos · Atul Bhaskar • Andy J. Keane
}

Received: date / Accepted: date

\begin{abstract}
This paper provides efficient and easy to implement formulations for two problems in structural optimization as second-order cone programming (SOCP) problems based on the minimum compliance method and derived using the principle of complementary en-
\end{abstract}

EPSRC grant EP/E004547/1

Early stages of the developments of this paper are provided in the recent conference paper of Makrodimopoulos et al (2008)

A. Makrodimopoulos (corresponding author)

School of Engineering Sciences, University of Southampton

Tel.: ++44-(0)2380-595194

Fax : ++44-(0)2380-594813

E-mail: am1a06@soton.ac.uk

\section{A. Bhaskar}

School of Engineering Sciences, University of Southampton

Tel.: ++44-(0)2380-593825

Fax : $++44-(0) 2380-594813$

E-mail: a.bhaskar@soton.ac.uk

\author{
A.J. Keane \\ School of Engineering Sciences, University of Southampton \\ Tel.: ++44-(0)2380-592944 \\ Fax : ++44-(0)2380-594813 \\ E-mail: andy.keane@soton.ac.uk
}

ergy. In truss optimization both single and multiple loads (where we optimize the worst-case compliance) are considered. By using a heuristic which is based on the SOCP duality we can consider a simple ground structure and add only the members which improve the compliance of the structure. It is also shown that thickness optimization is a problem similar to truss optimization. Examples are given to illustrate the method developed in this paper.

Keywords Structural optimization - Truss optimization - Thickness optimization - Second-order cone programming

\section{Introduction}

In this paper we take structural optimization to mean the arrangement of variables that define a structure (e.g. geometry, connectivity, cross sections) in order to get some optimal result, which can be minimum weight, 
compliance or stress and strain related quantities, depending on the problem being considered.

The optimal design of trusses, has been a very popular problem for more than a century (see e.g. Michell (1904)). This is due to the simplicity of designing with these structures and the ease in constructing mathematical models of them. Truss optimization consists of finding the optimal nodal positions, connectivities and cross section areas of a structure. Of course the optimal design of the truss is defined by the design goal, the most popular target being either the minimum weight of a rigid plastic structure (under single loading) or minimum compliance if the structure is made of elastic material. The reader can find a discussion of the similarities and differences in these problems in the works of Bendsøe et al (1994), Achtziger (1996) and Rozvany (2001).

The formulation of truss optimization with respect to all the parameters of a structure has a rather complicated form (see e.g. (Achtziger, 2007)) and, therefore, we cannot use techniques for standard optimization problems. The usual way to avoid including two of these parameters, the optimal positions of the nodes and their connectivities, is to consider a dense grid of nodes with all possible connections (as in Figure 1a) and solve the sizing optimization problem by mathematical programming techniques. In this way truss members with almost zero cross section area can be removed (see e.g. Kirsch (1989)). This approach was first suggested by Dorn et al (1964). As numerical optimiza- tion algorithms were rather weak in the early 90's, iterative procedures based on the optimality criteria such as those proposed by Rozvany and Zhou (1991); Zhou and Rozvany $(1991,1996)$ were preferred. For minimum compliance problems Ben-Tal and Bendsøe (1993) and Bendsøe et al (1994) developed two-level displacementbased approaches. Jarre et al (1998) suggested an interior point algorithm for problems with quadratic constraints. Also Kočvara and Outrata (2006) formulated the problem as a case of MPCC (Mathematical Program with Complementarity Constraints) considering large strains and displacements. An obvious drawback of approaches where we consider all possible connections is that a dense grid can result in a very large optimization problem. For this reason Achtziger (2007) experimented on approaches where the cross sections and the geometry of a truss (i.e. the nodal positions) are optimized simultaneously. In this way he avoided increasing the number of the truss members. Martinez et al (2007) suggested a "growth" method where new joints are added after each iteration. Another method in this class is given by Hagishita and Ohsaki (2007). On the other hand Gilbert and Tyas (2003) suggested that we can solve the sizing optimization problem for a simple ground structure (as in Figure 1b), and then detect which additional connectivities should be considered. In this way we have to solve a sequence of sizing problems, however each problem contains many fewer elements than if we consider all possible connections. 
They considered problems of plastic design for a single load case.

Thickness optimization can be considered as a case of sizing optimization for plane structures (i.e. plane stress, $2 \frac{1}{2} \mathrm{D}$ structures or plates). This problem is not as popular as truss optimization, however a lot of interest has been paid to the case of plates. A survey on this issue is given by Bendsøe (1995). Resizing algorithms on thickness optimization are given by Lam et al (2000). Thickness optimization can also be used as an intermediate step for topology problems, i.e. we can eliminate parts where the thickness tends to be zero (see e.g. Qing et al (2001)). Known procedures in this class of problems are ESO (e.g. Xie and Steven (1997)), NESO (Chen et al, 2002), the homogenisation method (Bendsøe and Kikuchi, 1988) and the formulation of Beckers (1999) which is relevant to discrete optimization.

A common issue for both problems (and all inequality problems in mechanics) is the formulation in a known form with inherent advantages. Such a case is the sizing optimization of trusses for plastic design: the problem can be formulated in terms of linear programming which is a relatively simple form of numerical optimization. Unfortunately this is not feasible in most cases, e.g. where sizing optimization has to be combined with topology optimization or with cases of varying loading. The use of general nonlinear programming (NLP) software can be a solution but this is not always most satisfactory. Although general NLP ${ }^{1}$ is very robust it is often not very efficient as it cannot take advantage of any special features of the optimization problem.

For thickness optimization problem, we also see that most of the procedures available in the literature, e.g. Qing et al (2001), Lam et al (2000) are iterative and not direct.

The present paper is concerned with the minimum compliance method. We provide second-order cone programming (SOCP) formulations for the two aforementioned problems and examine their effectiveness. A first flavor of such formulations in truss optimization was given by Ben-Tal and Nemirovski (1997) and Lobo et al (1998). In the first paper truss optimization was presented as a semi-definite programming problem which is a hyperset of SOCP. Later Ben-Tal and Nemirovski (2001) presented SOCP formulations derived from the principle of potential energy. Here we show that the use of the complementary energy can lead in a more straight forward manner to typical mathematical programming forms. The simplicity of using this principle becomes clearer when we present the formulation for the problem of thickness optimization. Here the formulations are derived for both single and varying loading (worst case compliance). For truss optimization, using the SOCP duality, even for the worst-case compliance

\footnotetext{
1 By general NLP we mean algorithms (or in fact matrix manipulations) which do not take into account the special structure of a problem. We do not mean the case of general nonlinear constraints.
} 
problem for multiple loading, we can extend the heuristic of Gilbert and Tyas (2003) and find optimal connectivities by starting from a simple ground structure. A common advantage in both problems, is the simplicity of the method because all we have to do is to calculate some elemental quantities, assemble them, and solve the resulting optimization problem by an effective algorithm. In the numerical examples we use the conic optimizer of MOSEK which is based on the algorithm of Andersen et al (2003). SOCP is a class of numerical optimization problems which has attracted the mathematical society and, therefore, even more efficient algorithms and software are expected to appear in future. This is likely to enhance the present work.

This paper is organised as follows. In the next section conic sets and their relevance to optimization are presented. In section 3 the truss optimization problem is cast as second-order cone programming problem. This is then extended to multiple load cases for worst case compliance. The problem of thickness optimization as an SOCP case is presented in section 4. Numerical implementations of the methods and the heuristic developed in sections 3 and 4 are presented with examples in section 5. Finally conclusions are drawn in section 6 .

\section{Conic sets and conic optimization}

A set $\mathcal{K}$ is called a cone if $\forall \mathbf{x} \in \mathcal{K}$ and $\lambda \geq 0, \lambda \mathbf{x} \in \mathcal{K}$. Its dual cone $\mathcal{K}^{*}$ is defined as

$\mathbf{x}^{T} \mathbf{y} \geq 0, \quad \forall \mathbf{x} \in \mathcal{K} \Leftrightarrow \mathbf{y} \in \mathcal{K}^{*}$
If $\mathcal{K}=\mathcal{K}^{*}$ then the cone is self-dual. For example, the set $\Re_{+}=\{x \mid x \geq 0\}$ is a self-dual cone, as is the quadratic (or second-order) cone, which has the form

$\mathcal{K}_{q}^{(n)}=\left\{\mathbf{x} \in \Re^{n} \mid x_{1} \geq\left\|\mathbf{x}_{2: n}\right\|, x_{1} \geq 0\right\}$

where $\|$.$\| is the Euclidean norm and \mathbf{x}_{2: n}=\left[x_{2} \ldots x_{n}\right]^{T}$. Another interesting self-dual example is the rotated quadratic cone

$\mathcal{K}_{r}^{(n)}=\left\{\mathbf{x} \in \Re^{n} \mid 2 x_{1} x_{2} \geq\left\|\mathbf{x}_{3: n}\right\|^{2}, x_{1}, x_{2} \geq 0\right\}$.

For brevity we may adopt the notation

$(y, z, \mathbf{x}) \in \mathcal{K}_{r}^{(n)}$

meaning that

$y, z \geq 0, \quad \mathbf{x} \in \Re^{n-2}$ and $2 y z \geq\|\mathbf{x}\|^{2}$.

Although this can readily be transformed into a standard quadratic cone of the form (2), it can also be treated directly in its original form, (Andersen et al, 2003). All the above are subsets of the semi-definite cone

$S_{+}^{(n)}=\left\{\mathbf{X} \in \Re^{n \times n} \mid \mathbf{X} \succeq 0, \quad X_{i j}=X_{j i}\right\}$.

A conic programming problem has the form

$$
\begin{array}{ll}
\min & \sum_{i=1}^{N} \mathbf{c}_{i}^{T} \mathbf{x}_{i}+\mathbf{c}_{\mathrm{f}}^{T} \mathbf{x}_{\mathrm{f}} \\
\text { s.t. } & \mathbf{x}_{i} \in \mathcal{K}_{i}, \quad \forall i \in\{1, \ldots, N\} \\
& \sum_{i=1}^{N} \mathbf{A}_{i} \mathbf{x}_{i}+\mathbf{A}_{\mathrm{f}} \mathbf{x}_{\mathrm{f}}=\mathbf{b}
\end{array}
$$

where $\mathbf{c}_{i}, \mathbf{x}_{i} \in \Re^{d_{i}}, \mathbf{c}_{\mathrm{f}}, \mathbf{x}_{\mathrm{f}} \in \Re^{n_{\mathrm{f}}}, \mathbf{A}_{i} \in \Re^{m \times d_{i}}, \mathbf{A}_{\mathrm{f}} \in$ $\Re^{m \times n_{\mathrm{f}}}, \mathbf{b} \in \Re^{m}$ and $\mathcal{K}_{i}$ are conic sets. The dual problem 

compliance problem reads as:

$$
\begin{array}{ll}
\max & \mathbf{b}^{T} \mathbf{y} \\
\text { s.t. } & \mathbf{s}_{i} \in \mathcal{K}_{i}^{*}, \quad \forall i \in\{1, \ldots, N\} \\
& \mathbf{A}_{i}^{T} \mathbf{y}+\mathbf{s}_{i}=\mathbf{c}_{i}, \forall i \in\{1, \ldots, N\} \\
& \mathbf{A}_{\mathrm{f}}^{T} \mathbf{y}=\mathbf{c}_{\mathrm{f}} .
\end{array}
$$

The type of the cone defines the specific type of the conic programming problem. In the case of linear, secondorder or semi-definite cones the problem is reduced to linear programming (LP), second-order cone programming (SOCP) and semi-definite programming (SDP) respectively. In all these cases $\mathcal{K}_{i}^{*}=\mathcal{K}_{i}$.

\section{Truss optimization}

3.1 The minimum compliance problem in truss optimization

Consider a truss structure with $N E$ members and $N U$ degrees of freedom. For a given volume of material $V$, our aim is to find the cross section areas of the bars such that the compliance will be minimized. It is generally more convenient to use the member volumes $\xi_{i}$ as the unknowns instead of the cross sectional areas of the members. For the sake of simplicity we shall consider that they are not bounded.

$$
\begin{array}{ll}
\min & \mathbf{p}^{T} \mathbf{u} \\
\text { s.t. } & \left(\sum_{i=1}^{N E} \xi_{i} \mathbf{K}_{i}\right) \mathbf{u}=\mathbf{p} \\
& \sum_{i=1}^{N E} \xi_{i}=V \\
& \xi_{i} \geq 0, \quad \forall i \in\{1, \ldots, N E\}
\end{array}
$$

where $\mathbf{p} \in \Re^{N U}$ and $\mathbf{u} \in \Re^{N U}$ are the load and the displacement vectors respectively. The first constraint represents the equilibrium, the second the limitation of the total volume, and the third the positiveness of the volume variables. For each member

$\mathbf{K}_{i}=\frac{E_{i}}{L_{i}^{2}} \mathbf{b}_{i} \mathbf{b}_{i}^{T}$

where $L_{i}, E_{i}$ are respectively the length and the Young's modulus of the $i$ th member. The column vector $\mathbf{b}_{i}$ relates the elongation along the axis of member $i$ with nodal displacement vector $\mathbf{u}$ so that

$e_{i}=\mathbf{b}_{i}^{T} \mathbf{u}$.

We notice that (9) is a non-convex problem, and therefore, the solution is rather difficult. For this reason alternative forms of this optimization problem have been presented (see e.g. Bendsøe et al (1994); Bendsøe (1995); Kočvara and Outrata (2006)). Engineering structures are often subjected to multiple loading cases. In these contexts, we are interested in calculating the "worstcase" compliance. Considering $m$ load cases the prob- 
lem reads:

$$
\begin{array}{ll}
\min _{\mathbf{u}, \boldsymbol{\xi}} \max _{j=1, \ldots, m} & \left(\mathbf{p}^{(j)}\right)^{T} \mathbf{u}^{(j)} \\
\text { s.t. } & \left(\sum_{i=1}^{N E} \xi_{i} \mathbf{K}_{i}\right) \mathbf{u}^{(j)}=\mathbf{p}^{(j)} \\
& \sum_{i=1}^{N E} \xi_{i}=V \\
& \xi_{i} \geq 0, \quad \forall i \in\{1, \ldots, N E\} .
\end{array}
$$

This problem is even more complicated than (9) because we have a case of two-level optimization. In most engineering structures the exact values of the applied loads are unknown, but we can assume that the loads vary arbitrarily within the range of a domain $\mathcal{L}$. In this case we have to solve a problem of the form

$$
\begin{array}{ll}
\min _{\mathbf{u}, \boldsymbol{\xi}} \max _{\mathbf{p}(t) \in \mathcal{L}} & \mathbf{p}^{T}(t) \mathbf{u}(t) \\
\text { s.t. } & \left(\sum_{i=1}^{N E} \xi_{i} \mathbf{K}_{i}\right) \mathbf{u}(t)=\mathbf{p}(t) \\
& \sum_{i=1}^{N E} \xi_{i}=V \\
& \xi_{i} \geq 0, \quad \forall i \in\{1, \ldots, N E\}
\end{array}
$$

where $t$ is a pseudo-time parameter.

In this section we will use the principle of the complementary energy - which gives the half of compliance - and also it will be shown that (9) and (12) can be formulated not only as a convex optimization problem but also as SOCP. The relationship between (13) and (12) will be discussed and it is shown in section 3.3 that (12) is a special case of (13).
3.2 Size optimization for trusses - single load case

A structure subjected to a specific load field will be in equilibrium if the complementary energy is minimized $\min \frac{1}{2} \int_{V} \boldsymbol{\sigma}^{T} \mathbf{C}^{-1} \boldsymbol{\sigma} d V$ s.t. $\boldsymbol{\sigma} \in S_{\mathrm{eq}}$

where the objective function is the complementary energy, $\boldsymbol{\sigma}$ are the physical stresses, $\mathbf{C}$ is the elasticity matrix and $S_{\text {eq }}$ is the set of the stresses which can carry the loads. Consider now the truss structure under a single load. Since the complementary energy is half of the compliance, the resulting optimization problem (see e.g. Bendsøe et al (1994)) now reads

$$
\begin{array}{ll}
\inf _{\boldsymbol{\xi}} \min _{\mathbf{q}} & \sum_{i=1}^{N E} \frac{L_{i}^{2}}{E_{i}} \frac{q_{i}^{2}}{2 \xi_{i}} \\
\text { s.t. } \quad & \mathbf{B q}=\mathbf{p} \\
& \sum_{i=1}^{N E} \xi_{i}=V \\
& \xi_{i}>0, \quad \forall i \in\{1, \ldots, N E\}
\end{array}
$$

where $\mathbf{q} \in \Re^{N E}$ is the internal axial forces vector and $\mathbf{B} \in \Re^{N U \times N E}, \quad \mathbf{B}=\left[\mathbf{b}_{1} \ldots \mathbf{b}_{N E}\right]$ is the equilibrium matrix. Problem (15) can be turned into a single minimization problem, and after setting $r_{i}=\frac{L_{i}^{2}}{E_{i}} \frac{q_{i}^{2}}{2 \xi_{i}}$ and all we have to do is to solve the following SOCP problem: $\begin{array}{ll}\min & \sum_{i=1}^{N} r_{i} \\ \text { s.t. } & \left(r_{i}, \xi_{i}, \bar{q}_{i}\right) \in \mathcal{K}_{r}^{(3)}, \forall i \in\{1, \ldots, N E\}\end{array}$

$$
\begin{aligned}
& \overline{\mathbf{B}} \overline{\mathbf{q}}=\mathbf{p} \\
& \sum_{i=1}^{N E} \xi_{i}=V
\end{aligned}
$$

where $\bar{q}_{i}=\frac{L_{i}}{\sqrt{E_{i}}} q_{i} \quad$ and $\quad \overline{\mathbf{B}}=\left[\overline{\mathbf{b}}_{1} \ldots \overline{\mathbf{b}}_{N E}\right]$ with $\overline{\mathbf{b}}_{i}=$ $\mathbf{b}_{i} \frac{\sqrt{E_{i}}}{L_{i}}$. 
The dual problem - after some manipulations - now reads as

$$
\begin{array}{ll}
\max & \mathbf{p}^{T} \mathbf{u}-z^{*} \\
\text { s.t. } & z^{*} \geq \frac{V}{2}\left(\overline{\mathbf{b}}_{i}^{T} \mathbf{u}\right)^{2}, \quad \forall i \in\{1, \ldots, N E\} \\
& z^{*} \geq 0
\end{array}
$$

or in more compact form

$$
\max _{\mathbf{u}}\left(\mathbf{p}^{T} \mathbf{u}-\frac{V}{2} \max _{i=1, \ldots, N E}\left(\overline{\mathbf{b}}_{i}^{T} \mathbf{u}\right)^{2}\right)
$$

The reason why the displacement vector appears is explained in the Appendix. An algorithm adapted for such "minmax" forms is given by Bendsøe et al (1994). Also Jarre et al (1998) investigated this special form. An interesting issue is that the problem can be reduced even further as a linear programming case. However this can occur only if we do not consider other restrictions such as fixed values or upper bounds for the volume members. These issues and the analogy of the minimum compliance problem with the plastic design have been reported and analysed by Achtziger (1996) and Bendsøe et al (1994). A reduction to linear programming is also given in the book of Ben-Tal and Nemirovski (2001).

\subsection{Sizing optimization of trusses for the worst-case} compliance

We consider here that the structure is subjected to arbitrarily varying loads within a load domain $\mathcal{L}$. By applying the principle of complementary energy the opti- mization problem reads:

$$
\begin{array}{ll}
\min _{\xi, \mathbf{q}} \max _{t \in T} & \sum_{i=1}^{N E} \frac{L_{i}^{2}}{E_{i}} \frac{q_{i}^{2}(t)}{2 \xi_{i}} \\
\text { s.t. } \quad & \mathbf{B q}(t)=\mathbf{p}(t) \\
& \sum_{i=1}^{N} \xi_{i}=V \\
& \xi_{i}>0, \quad \forall i \in\{1, \ldots, N E\}
\end{array}
$$

where $t$ is the pseudo-time parameter such that $\mathbf{p}(t) \in$ $\mathcal{L}$ and $T$ is a set such that $T=\{t: \mathbf{p}(t) \in \mathcal{L}\}$. The worst compliance can be represented by a variable

$r^{*} \geq \sum_{i=1}^{N E} r_{i}(t), \quad \forall t \in T$

where $r_{i}(t) \geq \frac{L_{i}^{2}}{E_{i}} \frac{q_{i}^{2}(t)}{2 \xi_{i}} \quad \forall t \in T, \quad \forall i \in\{1, \ldots, N E\}$.

The latter inequality can be rewritten as

$2 r_{i}(t) \xi_{i} \geq \frac{L_{i}^{2}}{E_{i}} q_{i}^{2}(t)$

which, in fact, is a rotated quadratic cone. Now the problem takes the form

$\min r^{*}$

$$
\begin{aligned}
& \text { s.t. }\left(r_{i}(t), \xi_{i}, \bar{q}_{i}(t)\right) \in \mathcal{K}_{r}^{(3)}, \forall i \in\{1, \ldots, N E\}, \forall t \in T \\
& \sum_{i=1}^{N E} r_{i}(t)-r^{*} \leq 0, \quad \forall t \in T \\
& \overline{\mathbf{B}} \overline{\mathbf{q}}(t)=\mathbf{p}(t), \quad \forall t \in T \\
& \sum_{i=1}^{N E} \xi_{i}=V
\end{aligned}
$$

where $\bar{q}_{i}, \overline{\mathbf{B}}$ are as defined previously after equation (16). An important issue is how to eliminate the variable $t$ and apply the constraints for finite times. It is reasonable to assume that the vector $\mathbf{p}(t)$ is a linear combination of $N L$ load cases i.e.

$\mathbf{p}(t)=\sum_{j=1}^{N L} \mu_{j}(t) \mathbf{p}^{(j)}$ 
where the $\mu_{j}$ factors are defined by a set of linear inequalities $\mathbf{A} \boldsymbol{\mu} \leq \mathbf{d}$. We assume that $\mathcal{L}$ is a convex hyperpolyhedron with $N V$ vertices. This implies that the load vector can also be written in the form

$\mathbf{p}(t)=\sum_{i=1}^{N V} \gamma_{j}(t) \mathbf{p}^{(j)}$

with $\sum_{j=1}^{N V} \gamma_{j}(t)=1$ and $\gamma_{j}(t) \geq 0$. As a result a similar relation would hold for $\overline{\mathbf{q}}(t)$. Also we can assume that $\left[\begin{array}{c}r_{i}(t) \\ \xi_{i} \\ \bar{q}_{i}(t)\end{array}\right]=\sum_{j=1}^{N V} \gamma_{j}(t)\left[\begin{array}{c}\bar{r}_{i}^{(j)}(t) \\ \xi_{i} \\ \bar{q}_{i}^{(j)}(t)\end{array}\right]$.

where $\bar{r}_{i}^{j} \geq \frac{L_{i}^{2}}{E_{i}} \frac{\left(\bar{q}_{i}^{(j)}\right)^{2}}{2 \xi_{i}}$. This means that it is sufficient to apply inequality (21) for the quantities which correspond to the vertices of the domain $\mathcal{L}$. Also because we want a variable to belong to only one cone we introduce the auxiliary constraints

$\bar{\xi}_{i}^{(j)}=\xi_{i}, \quad \forall i \in\{1, \ldots, N E\}, \quad \forall j \in\{1, \ldots, N V\}$

where $j$ represents the indices of the load domain and for conciseness we introduced the sets

$I_{E}=\{1, \ldots, N E\} \quad$ and $\quad J_{V}=\{1, \ldots, N V\}$.

Therefore the problem takes the form

$\min r^{*}$

$$
\begin{aligned}
& \text { s.t. }\left(\bar{r}_{i}^{(j)}, \bar{\xi}_{i}^{(j)}, \bar{q}_{i}^{(j)}\right) \in \mathcal{K}_{r}^{(3)}, \forall i \in I_{E}, \quad \forall j \in J_{V} \\
& \sum_{i=1}^{N E} \bar{r}_{i}^{(j)}-r^{*} \leq 0, \quad \forall j \in J_{V} \\
& \overline{\mathbf{B}} \overline{\mathbf{q}}_{i}^{(j)}=\mathbf{p}^{(j)}, \quad \forall j \in J_{V} \\
& \bar{\xi}_{i}^{(j)}=\xi_{i}, \quad \forall i \in I_{E}, \quad \forall j \in J_{V} \\
& \sum_{i=1}^{N E} \xi_{i}=V \text {. }
\end{aligned}
$$

The following general remarks about this formulation should be made:

- Only the worst-case compliance is minimized. Other compliances do not necessarily correspond to the minimum complementary energy for the calculated volumes and, therefore, the stresses would not correspond to their respective load cases.

- In the case of specific finite load cases i.e. $\mathbf{p}_{1}, \ldots, \mathbf{p}_{N}$ this problem corresponds to the worst compliance for a load domain whose load vector is given by

$\mathbf{p}(t)=\sum_{j=1}^{N} \gamma_{j}(t) \mathbf{p}^{(j)}+\gamma_{0}(t) \mathbf{p}^{(0)}$

with $\sum_{j=0}^{N} \gamma_{j}(t)=1, \quad \gamma_{j}(t) \geq 0$ and $\mathbf{p}^{(0)}=\mathbf{0}$, i.e. the load domain has the form of a hyper-triangle of $N+1$ load vertices. This case is also referred to as alternating loading and this is the usual way that multiple load cases are considered.

- Consider minimizing the compliance for each load load vector $\mathbf{p}^{(j)}$. The lowest compliance arises, say, for the case $j=k$ which gives a compliance $\Pi^{*}$ for $\boldsymbol{\xi}=\boldsymbol{\xi}^{*}$. If, for this volume vector, the compliance for the other load cases is still lower than $\Pi^{*}$ the result for the single load case $\mathbf{p}^{(k)}$ will be the same as for the worst-case compliance. 
3.4 A heuristic for adding new members

The dual problem of (28) - see duality between (7) and

(8) - will have the form

$$
\begin{array}{lll}
\max & \sum_{j=1}^{N V}\left(\mathbf{p}^{(j)}\right)^{T} \mathbf{u}^{(j)}+V z & \\
\text { s.t. } & \left(\psi_{i}^{(j)}, \phi_{i}^{(j)}, \tilde{e}_{i}^{(j)}\right) \in \mathcal{K}_{r}^{(3)}, \forall i \in I_{E}, \quad \forall j \in J_{V} \\
& \overline{\mathbf{B}}^{T} \mathbf{u}^{(j)}+\tilde{\mathbf{e}}^{(j)}=\mathbf{0}, \quad \forall j \in J_{V} & \\
& \bar{\psi}^{(j)}+\psi_{i}^{(j)}=0, & \forall i \in I_{E}, \quad \forall j \in J_{V} \\
& \bar{\phi}_{i}^{(j)}+\phi_{i}^{(j)}=0, & \forall i \in I_{E}, \quad \forall j \in J_{V} \\
& -\sum_{j=1}^{N V} \bar{\phi}_{i}^{(j)}+z=0, & \\
& \\
& -\sum_{j=1}^{N V} \bar{\psi}^{(j)}=1 &
\end{array}
$$

where $z \in \Re$ and $\tilde{\mathbf{e}}^{(j)} \in \Re^{N E}$. After setting $z^{*}=-V z$ the problem can be reduced to

$$
\begin{aligned}
\max & \sum_{j=1}^{N V}\left(\mathbf{p}^{(j)}\right)^{T} \mathbf{u}^{(j)}-z^{*} \\
\text { s.t. } & \bar{\psi}^{*(j)} \phi_{i}^{(j)} \geq \frac{V}{2}\left(\overline{\mathbf{b}}_{i}^{T} \mathbf{u}^{(j)}\right)^{2}, \forall i \in I_{E}, \quad \forall j \in J_{V} \\
& \sum_{j=1}^{N V} \phi_{i}^{(j)}=z^{*}, \quad \forall i \in I_{E} \\
& \sum_{j=1}^{N V} \bar{\psi}^{*(j)}=1 .
\end{aligned}
$$

Now say that we connect two nodes that are existing but unconnected. By connecting them we have a new optimization problem and the question is whether this will lead to lower compliance. This will not occur if for the existing nodal solutions the set defined by the constraints remains feasible. That is by adding a member, the dimension of the $\mathbf{u}$ and $\bar{\psi}^{*(j)}$ variables will remain the same. Now if we form the vector $\mathbf{b}$ and we have the
This is because we can increase the values of $\phi^{(j)}$ variables as much as we need in order to turn the inequality to strict equality. Therefore a necessary (though not sufficient) condition that the added member will reduce

additional constraints

$$
\begin{aligned}
& \phi^{(j)} \geq V \frac{\left(\overline{\mathbf{b}}^{T} \mathbf{u}^{(j)}\right)^{2}}{2 \bar{\psi}^{*(j)}} \\
& \sum_{j=1}^{N V} \phi_{i}^{(j)}=z^{*}
\end{aligned}
$$

Assuming that we give to $\mathbf{u}$ and $\bar{\psi}^{*(j)}$ the same values then the problem will remain feasible if for $\phi_{i}^{(j)}=$ $V \frac{\left(\overline{\mathbf{b}}^{T} \mathbf{u}^{(j)}\right)^{2}}{2 \bar{\psi}^{*(j)}}$

$$
\sum_{j=1}^{N V} \phi_{i}^{(j)} \leq z^{*}
$$

the compliance is that (34) must not hold i.e. it should be

$\sum_{j=1}^{N V} \phi_{i}^{(j)}>z^{*}$.

In this way, we can start with a simple ground structure (meaning that we do not consider all necessary connections), solve (28) and check if additional members could be added. If yes the procedure is repeated. For the case of single loading this condition becomes

$$
\frac{V}{2}\left(\overline{\mathbf{b}}^{T} \mathbf{u}\right)^{2}>z^{*}
$$

The procedure is summarized in Table 1 . We should point out that both the values of the variables of the primal and the dual problem are given when we use most of the optimization software.

Because the number of the additional potential members may be too high we select only some of them. 
Therefore from the candidate members, we select those which violate the condition (34) most. Also we should take care that the number of added members is not too small. In this way we avoid having to repeat solving too many sizing optimization problems. The procedure is given in Table 2. Finally we exclude the addition of collinear members and we consider the limitation $L<2 L_{\max }$

\section{Thickness optimization in plane stress as SOCP}

Thickness optimization is also an interesting problem and has been applied to plates (for a detailed literature review see Bendsøe (1995)) and also plane stress (see e.g. Qing et al (2001)). For a given volume we have to find the best thickness field $h(x, y)$ which would result in the optimum compliance. Using the principle of minimization of the complementary energy we get

$\min \frac{1}{2} \int_{V} \boldsymbol{\sigma}^{T} \mathbf{C}^{-1} \boldsymbol{\sigma} h d A$

s.t. $\boldsymbol{\sigma} \in S_{\mathrm{eq}}$

$$
\begin{aligned}
& \int_{A} h d A=V \\
& h(x, y) \geq 0
\end{aligned}
$$

where the first integral in the objective represents the complementary energy and the integral in the constraints represents the total volume. We also require the thickness field to be non-negative. Now if the structure is discretized into $N E$ displacement finite elements the problem takes the form

$$
\begin{aligned}
\min & \sum_{i=1}^{N E} \int_{A_{e}} \boldsymbol{\sigma}^{T} \mathbf{C}^{-1} \boldsymbol{\sigma} h d A_{i} \\
\text { s.t. } & \sum_{i=1}^{N E} \int_{A_{e}} \mathbf{H}^{T} \boldsymbol{\sigma} h d A_{i}=\mathbf{p} \\
& \sum_{i=1}^{N E} \int_{A_{e}} h d A_{i}=V \\
& h(x, y) \geq 0
\end{aligned}
$$

where $\mathbf{H}$ is the strain displacement matrix. Assume now that we use in total $N G$ number of Gauss points in order to calculate the integrals. Then we get

$$
\begin{aligned}
\min & \frac{1}{2} \sum_{i=1}^{N G} \boldsymbol{\sigma}_{i}^{T} \mathbf{C}^{-1} \boldsymbol{\sigma}_{i} h_{i} w_{i} \\
\text { s.t. } & \sum_{i=1}^{N G} \mathbf{H}_{i}^{T} \boldsymbol{\sigma}_{i} w_{i} h_{i}=\mathbf{p} \\
& \sum_{i=1}^{N G} h_{i} w_{i}=V \\
& h_{i} \geq 0
\end{aligned}
$$

where $w_{i}$ is the weight associated with the $i$ th Gauss point and the determinant of the Jacobian between the global coordinates and the physical ones. If we set

$\xi_{i}=w_{i} h_{i} \quad$ and $\quad \overline{\boldsymbol{\sigma}}_{i}=\boldsymbol{\sigma}_{i} h_{i} w_{i}$

the problem takes the form

$$
\begin{aligned}
\min & \sum_{i=1}^{N G} \frac{\overline{\boldsymbol{\sigma}}_{i}^{T} \mathbf{C}^{-1} \overline{\boldsymbol{\sigma}}_{i}}{2 \xi_{i}} \\
\text { s.t. } & \sum_{i=1}^{N G} \mathbf{H}_{i}^{T} \overline{\boldsymbol{\sigma}}_{i}=\mathbf{p} \\
& \sum_{i=1}^{N G} \xi_{i}=V \\
& \xi_{i} \geq 0
\end{aligned}
$$

which is very similar to the problem of truss optimization (e.g compare with equation (15)). Each Gauss point, for the thickness optimization problem, is analogue to a truss member, the scaled stress vector $\overline{\boldsymbol{\sigma}}_{i}$ is analogous to the axial forces, the $\xi_{i}$ quantities are similar to 
the volume of the members. The matrix $\mathbf{C}$ is positive definite and, therefore, we can write

$\mathbf{C}^{-1}=\mathbf{Q}^{T} \mathbf{Q}$

and transform the stresses at the $i$ th Gauss point to a new variable $\mathbf{z}_{i}=\mathbf{Q} \overline{\boldsymbol{\sigma}}_{i}$. We also set

$r_{i}=\frac{\|\mathbf{Q} \overline{\boldsymbol{\sigma}}\|^{2}}{2 \xi_{i}}$.

In terms of the new variables $\mathbf{z}$ the problem takes the form

$\min \sum_{i=1}^{N G} r_{i}$

s.t. $\left(\xi_{i}, r_{i}, \mathbf{z}_{i}\right) \in \mathcal{K}_{r}^{(5)}, \quad \forall i \in\{1, \ldots, N G\}$

$$
\begin{aligned}
& \sum_{i=1}^{N G}\left(\mathbf{H}_{i}^{T} \mathbf{Q}^{-1}\right) \mathbf{z}_{i}=\mathbf{p} \\
& \sum_{i=1}^{N G} \xi_{i}=V .
\end{aligned}
$$

For the case of a convex linearized convex load domain, the worst-case compliance problem will have the form

$\min r^{*}$

$$
\begin{array}{lll}
\text { s.t. } & \left.\bar{r}_{i}^{(j)}, \xi_{i}, \mathbf{z}_{i}^{(j)}\right) \in \mathcal{K}_{r}^{(5)}, \quad \forall i \in I_{G}, \forall j \in J_{V} \\
& \sum_{i=1}^{N G} \bar{r}_{i}^{(j)} \leq r^{*}, \quad \forall j \in J_{V} \\
& \sum_{i=1}^{N G}\left(\mathbf{H}_{i}^{T} \mathbf{Q}^{-1}\right) \mathbf{z}_{i}^{(j)}=\mathbf{p}^{(j)}, \forall j \in J_{V} \\
& \sum_{i=1}^{N G} \xi_{i}=V
\end{array}
$$

where $I_{G}=\{1, \ldots, N G\}$.

Note that regarding thickness several other different schemes (e.g. continuous thickness) could be employed. However the technique for the formulation as an SOCP problem - which is the main aim of this paper - would be the same.

\section{Numerical implementations}

Having transformed the two problems being considered into SOCP forms, we used the MOSEK (MOSEK ApS, 2007) SOCP optimizer for numerical implementation. The optimizer is based on the algorithm which was presented by Andersen et al (2003). Other standard SOCP software that could be used are SeDuMi (Sturm, 1999), SDPT3 (Tütüncü et al, 2003) and PENNON (Kočvara and Stingl, 2003), for example. For the optimization procedure we deactivated the function concerning the detection of linear dependencies within MOSEK. Also, the commercial software GiD (CIMNE, 2007) was used as a pre/post-processor during thickness optimization. The analyses were performed on a PC with a $2.40 \mathrm{GHz}$ Intel Core 2 Duo CPU, and 3 GB RAM in the Windows $\mathrm{XP}$ environment.

\subsection{Truss optimization of a supported structure}

We consider the structure shown in Figure 2 which has also been examined by Bendsøe et al (1994). As in Bendsøe et al (1994), the cases of single and alternate loading are examined. All loads take the same value $P$ when applied. First we applied the sizing optimization for all possible connections and for grids of $13 \times 13$ and $25 \times 25$ nodes. The results and statistics are given in Table 3 . The compliance is multiplied by $E V / P^{2} L^{2}$ and is given in the $W$ column. Next we applied the heuristic of starting with a ground structure as in Figure $1 \mathrm{~b}$ and adding members following the procedure presented in 
Table 2. The heuristic was applied for the same grids but also for a grid of $37 \times 37$ nodes. The resulting statistics are given in Tables 4,5. Having seen that the upper half of the structure is inactive (ie. no members of this part belong to the optimal layout) we considered a domain of $L / 2$ height and a Grid of $49 \times 97$. Results and statistics are given in Table 6 .

Optimal layouts are given in Figures 3,4,5 and 6. We observe that the optimal layouts for the $13 \times 13$ grid are the same as in Bendsøe et al (1994). Note that for the same grid it is computationally less time consuming to start with a simple ground structure and add members than to solve the problem of all possible connections. For the $25 \times 25$ grid problem and the single loading case, just $2.0 \mathrm{sec}$ is needed for the method proposed in this paper whereas $42 \mathrm{sec}$ are needed if we solve the problem with all connections (as in Figure 1a). Moreover a problem with an excessive number of bars may not be solvable due to memory limitations or the weakness of the applied algorithm to converge for an extremely large problem. This is a further advantage of the sequential procedure. The following general observations can be made:

- In the various layouts given in Figure 3 we see that very often active members become inactive and vice versa. This is not necessarily related to the number of the added members. Specially for the single load case, although very few members were added after the third stage and the compliance was reduced by $1 \%$, there is a clear difference between the quality of the solutions of these last four stages. This means that little difference between the values of the compliances does not mean that there will be strong similarity in the values of the design parameters (in our case the cross section areas).

- The first few steps seem to be sufficient to get a very good approximation of the optimal compliance.

- The solution of problems with multiple loading takes significantly more time. This feature can be improved if we customise the optimizer so that it can take advantage of the angular form of the matrix data. The optimizer behaved very well. One exception is the last stage of the $37 \times 37$ grid (single loading case), where there was a slight instability. The optimizer ended in a "near optimal" status. However the error is limited to the last digit (the sixth) of the objective function and the tolerances were very close to those when it terminates normally.

\subsection{Truss optimization for a cantilever beam}

Consider the cantilever beam of Figure 7 . The worstcase compliance optimization method is applied for the following cases:

- Case I; $P_{1}=2 P$ and $P_{2}=P$ (single loading)

- Case II; the loads $P_{1}$ and $P_{2}$ vary in a way such that $-2 P \leq P_{1} \leq 2 P \quad$ and $\quad 0 \leq P_{2} \leq P$

- Case III; the loads are applied alternatively in a way such that

$P_{1}=2 P \quad$ and $\quad P_{2}=P$ 
- Case IV; $P_{1}=-2 P$ and $P_{2}=P$ (single loading)

- Case V; the loads $P_{1}$ and $P_{2}$ vary in a way such that

$-2.02 P \leq P_{1} \leq-1.98 P \quad$ and $\quad 0.99 P \leq P_{2} \leq 1.01 P$

The positive sign means that the load has the direction shown in the figure. A grid of size $21 \times 41$ was considered. In all analyses we started with a ground structure like the one in Figure $1 \mathrm{~b}$ and we added members based on the heuristic in Table 1. The optimal layouts are shown in Figure 8. Cases I and II gave the same layouts and compliance. It is interesting that the layout looks as if we constructed two separate layouts. We also notice that the layout of case III is complicated although the shape of the "thick part" is a bit clearer. Case IV is of particular interest. We notice that only one joint appears to be part of the optimal layout which means that the beam is not supported. However this layout is reasonable if we take into account that the total moment at the area of the support is zero. Case $\mathrm{V}$ is nothing but the worst case of a perturbation of the load values of case IV by $\pm 1 \%$. Indeed we see that more than one member is connected with the support area. Some statistics are given in Table 7 . In all cases the number of optimization problems that had to be solved was almost the same.

\subsection{Thickness optimization examples}

The four plane stress structures in Figure 9 were explored for thickness optimization. Here we set $L / H=2$.
We applied thickness optimization for different meshes. Each mesh consisted of $M \times N$ quadrants and each of them was subdivided into four six-node triangular elements. For each element, six Gauss points were used for numerical integration. This means that each element involves 18 stress variables. Data concerning the optimization procedure and the compliance are given in Table 8. The compliance is multiplied by $E V / P^{2} L^{2}$ and is given in the $W$ column. It can be seen that, between different meshes, there is little difference in the values of the optimal compliance. We also note from Figure 10 that between two meshes:

- The general scheme of the contour of the optimal thickness is similar;

- Although the difference between the compliances is very little, the value of the maximum thickness is increased. In fact it is doubled.

The same structures were discretized as trusses and sizing optimization was applied for grids of $21 \times 41$ nodes. We compare their optimal layouts in Figure 11 with the optimal thickness in Figure 10. Concerning the structures A and B (see Figure 9) we see that thickness contours reveal quite clearly the boundaries of the optimal structures but not internal parts. Concerning structure $\mathrm{C}$ we see that boundaries of the central part are revealed quite well, however that left and right parts do not look like truss members. Also the compliance of the truss structures A,B and C was $10 \%$ higher than the corresponding continuous structure. Concerning struc- 
ture $\mathrm{D}$ we see that both the layout and the compliance were almost identical.

Thickness optimization gives layouts which are similar to those of the homogenisation method for the rank2 layering (Bendsøe (1995), page 35).We also note that the resulting optimization problems were solved fairly rapidly even though they contained hundred of thousands of stress variables (e.g. the $40 \times 80$ mesh involves 230400 stress variables). This is due to the sparsity of the matrix data which endorses the use of algorithms based on the interior point method.

\section{Conclusions}

In this paper, by using the principle of complementary energy, it is shown that truss and thickness optimization in terms of the minimum compliance method can be formulated as SOCP problems. This is advantageous since several standard SOCP solvers are available and, therefore, the proposed methods can be easily implemented. Practicing engineers need only to calculate elemental matrix data and solve the resulting optimization problem. Another advantage of the proposed method is the efficiency and the speed of the optimizer which was used.

Concerning truss optimization, the simplicity of the structure of SOCP allowed us to exploit duality even for the worst-case compliance problem, and modify-extend the heuristic of Gilbert and Tyas (2003) on adding members. In this way we solve a sequence of finite ele- ment runs involving many fewer elements than using all possible connections. Note that the usefulness of this heuristic has nothing to do with the algorithm that we use, nor even its efficiency. Whatever the efficiency of the optimization software used (and whether this is NLP or SOCP) the heuristic is introduced in order to solve problems with even denser grids.

In the case of thickness optimization, it was shown that we can obtain results similar to those of the homogenization method using rank-2 layering. This means that thickness optimization identifies boundaries but not "internal structures". Another similarity is that thickness plays a role similar to material density, however without being subjected to limitations induced by the microstructure. The fact that it is formulated as an SOCP case is an advantage compared to homogenization which leads to non-linear programming problems.

\section{Appendix}

Compliance can be found by using the principle of the minimum potential energy:

$$
\begin{array}{cc}
\min _{\boldsymbol{\xi}} \max _{\mathbf{u}} & \mathbf{p}^{T} \mathbf{u}-\frac{1}{2} \sum_{i=1}^{N E} \frac{E_{i} \xi_{i}}{L_{i}^{2}}\left(\mathbf{b}_{i}^{T} \mathbf{u}\right)^{2} \\
\text { s.t. } & \sum_{i=1}^{N E} \xi_{i}=V \\
& \xi_{i} \geq 0
\end{array}
$$

However for a given displacement vector the quantity will be minimized if the second part is maximized because $\frac{E_{i}}{L_{i}^{2}}\left(\mathbf{b}_{i}^{T} \mathbf{u}\right)^{2} \geq 0$. Maximization occurs for the element which gives the highest quantity multiplied by 
$\xi_{i}=V$. Therefore, the problem (after taking into account the definition of $\overline{\mathbf{b}}_{i}$ ) becomes equivalent to

$\max _{\mathbf{u}}\left\{\mathbf{p}^{T} \mathbf{u}-\frac{V}{2} \max _{i=1, \ldots, N E}\left(\mathbf{b}_{i}^{T} \mathbf{u}\right)^{2}\right\}$

\section{References}

Achtziger W (1996) Truss topology optimization including bar properties different for tension and compression. Structural and Multidisciplinary Optimization 12:63-74

Achtziger W (2007) On simultaneous optimization of truss geometry and topology. Structural and Multidisciplinary Optimization 33:285-304

Andersen E, Roos C, Terlaky T (2003) On implementing a primal-dual interior-point method for conic quadratic optimization. Mathematical Programming Series B 95:249-277

Beckers M (1999) Topology optimization using a dual method with discrete variables. Structural Optimization $17: 14-24$

Ben-Tal A, Bendsøe M (1993) A new method for optimal truss design topology design. Siam Journal on Optimization 3:322-358

Ben-Tal A, Nemirovski A (1997) Robust truss topology design via semidefinite programming. Siam Journal on Optimization 7:991-1016

Ben-Tal A, Nemirovski A (2001) Lectures on modern convex optimization. MPS-SIAM Series on Optimization, Philadelphia
Bendsøe M (1995) Optimization of Structural Topology, Shape and Material. Springer-Verlag, Berlin

Bendsøe M, Kikuchi N (1988) Generating optimal topologies in structural design using a homogenization method. Computer Methods in Applied Mechanics and Engineering 71:197-224

Bendsøe M, Ben-Tal A, Zowe J (1994) Optimization methods for truss geometry and topology design. Structural Optimization 7:141-159

Chen YM, Bhaskar A, Keane A (2002) A parallel nodal-based evolutionary structural optimization algorithm. Structural and Multidisciplinary Optimization 23:241-251

CIMNE (2007) GiD Version 8.0. Reference Manual

Dorn W, Gomory R, Greenberg H (1964) Automatic design of optimal structures. J de Mechanique 3:2552

Gilbert M, Tyas A (2003) Layout optimization of largescale pin jointed frames. Engineering Computations 20:1044-1064

Hagishita T, Ohsaki M (2007) Topology optimization of trusses by growing ground structure method. Structural and Multidisciplinary Optimization, in press.

Jarre F, Kočvara M, Zowe J (1998) Optimal truss design by interior point methods. Siam Journal on Optimization 8:1084-1117

Kirsch U (1989) Optimal topologies of truss structures. Computer Methods in Applied Mechanics and Engineering 72:15-28 
Kočvara M, Outrata J (2006) Effective reformulations of the truss topology design problem. Optimization and Engineering 7:201-219

Kočvara M, Stingl M (2003) Pennon a code for convex nonlinear and semidefinite programming. Optimization Methods and Software 18:317-333

Lam Y, Manickarajah D, Bertolini A (2000) Effective reformulations of the truss topology design problem. Finite Elements in Analysis and Design 34:159-174

Lobo M, Vandenberghe L, Boyd S, Lebret H (1998) Applications of second-order cone programming. Linear Algebra and its applications 284:193-228

Makrodimopoulos A, Bhaskar A, Keane A (2008) A formulation for large-scale truss optimization. In: CDROM PROCEEDINGS of the 6th GRACM International Congress on Computational Mechanics, Thessaloniki, 19-21 June

Martinez P, Marti P, Querin O (2007) Growth method for size, topology, and geometry optimization of truss structures. Structural and Multidisciplinary Optimization 33:13-26

Michell A (1904) The limits of economy of material in frame-structures. Philosophical Magazine 8:589-597

MOSEK ApS (2007) The MOSEK optimization tools manual. Version 5.0, Revision 60

Qing L, GP GS, Xie Y (2001) Evolutionary thickness design with stiffness maximization and stress minimization criteria. International Journal for Numerical Methods in Engineering 52:979-995
Rozvany G (2001) Stress ratio and compliance based methods in topology optimization-a critical review. Structural and Multidisciplinary Optimization 21:109-119

Rozvany G, Zhou M (1991) The COC algorithm, Part I: Cross-section optimization or sizing. Computer Methods in Applied Mechanics and Engineering 89:281-308

Sturm J (1999) Using sedumi 1.02, a matlab toolbox for optimization over symmetric cones. Optimization Methods and Software 11-12:625-653

Tütüncü R, Toh K, Todd M (2003) Solving semidefinite-quadratic-linear programs using SDPT3. Mathematical Programming 95:189-217

Xie Y, Steven G (1997) Evolutionary structural optimization. Springer-Verlag, Berlin

Zhou M, Rozvany G (1991) The COC algorithm, Part II: Topological, geometrical and generalized shape optimization. Computer Methods in Applied Mechanics and Engineering 89:309-336

Zhou M, Rozvany G (1996) An improved approximation technique for the dcoc method of sizing optimization. Computers and Structures 60:763-769 
Table 1 Heuristic procedure

\begin{tabular}{|c|c|}
\hline Step 1 & Consider an initial structure \\
\hline Step 2 & Construct the numerical data for the problem (28) \\
\hline Step 3 & Solve (28) and obtain $\boldsymbol{\xi}, z^{*}, \mathbf{u}^{(j)}, \bar{\psi}^{*(j)}, \quad j=1, \ldots, N V$ \\
\hline Step 4 & Set counter $=0$ \\
\hline Step 5 & For each pair of non-connected nodes: \\
\hline Step 5.1 & construct the vector $\overline{\mathbf{b}}$ \\
\hline \multirow[t]{2}{*}{ Step 5.2} & for each load vertex calculate the quantity \\
\hline & $\phi^{(j)}=V\left(\overline{\mathbf{b}}^{T} \mathbf{u}^{(j)}\right)^{2} / 2 \bar{\psi}^{*(j)}$ \\
\hline \multirow[t]{2}{*}{ Step 5.3} & if $\sum_{j=1}^{N V} \phi^{(j)}>z^{*}$ \\
\hline & accept the new connection and set counter $=$ counter +1 \\
\hline \multirow[t]{2}{*}{ Step 6} & if counter $=0$ then Stop \\
\hline & if counter $>0$ goto Step 2 including the new connections as well \\
\hline
\end{tabular}


Table 2 The procedure of Table1 modified

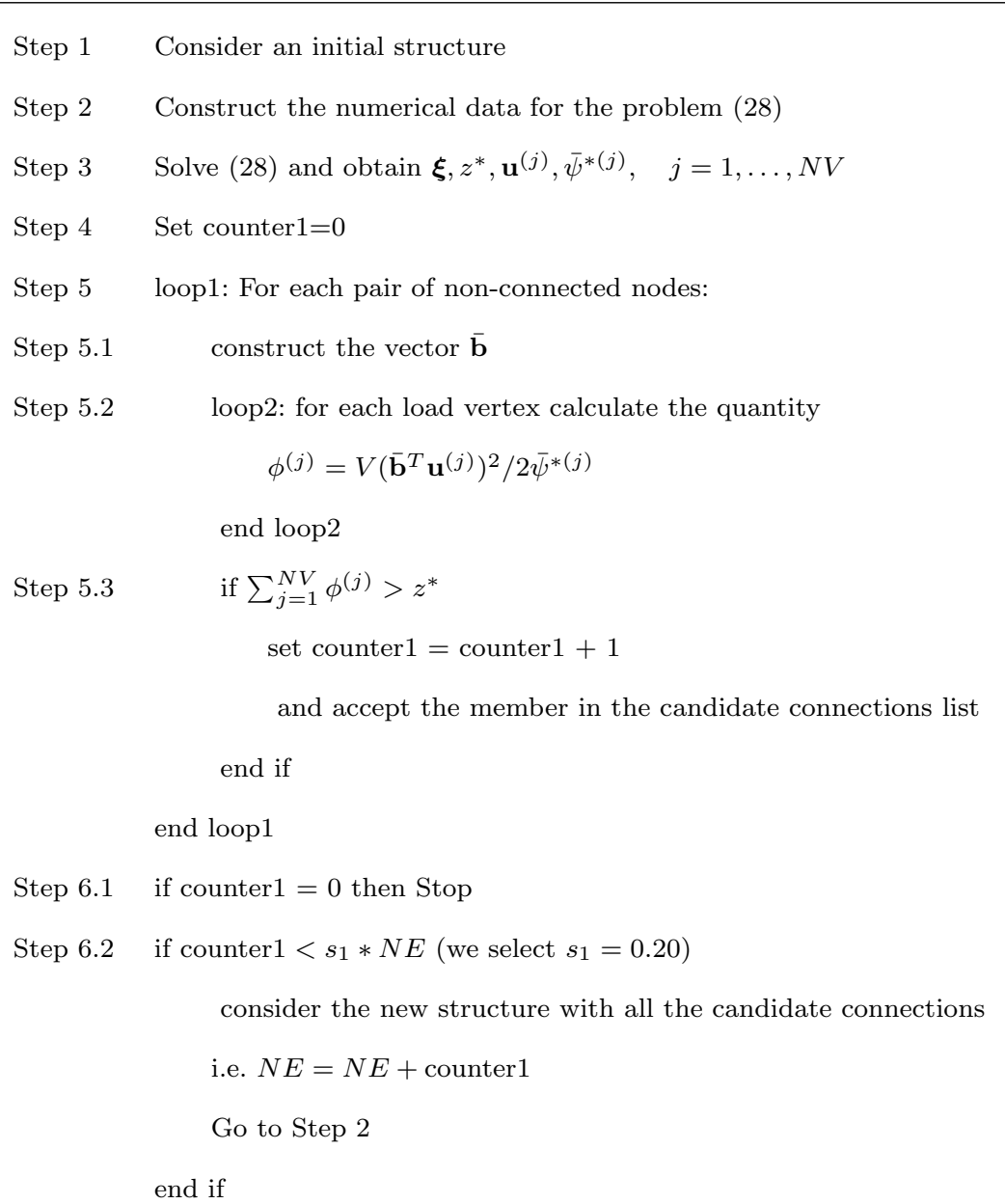

Step 6.3 loop1: for each of the candidate members

calculate the quantity $\beta_{i}=\sum_{j=1}^{\text {counter } 1} \phi^{(j)}-z^{*}$

end loop1

Step 6.4 calculate $\beta_{\max }=\max _{i=1, \text { counter } 1} \beta_{i}$

set counter $2=0$ and $s_{2}=\min \{0.50$, counter $1 / N E\}$

loop1:for each member with $\beta_{i}>s_{2} * \beta_{\max }$

set counter $2=$ counter $2+1$

end loop1

if counter $2<s_{2} * N E$

consider the new structure with $N E=N E+$ counter 2 elements

Go to Step 2

else

add the members with the $s_{1} * N E$ higher values of $\beta_{i}$

and consider the new structure with $N E=N E+s_{1} * N E$

Go to Step 2

end if 
Table 3 Results and statistics for all possible connections for the structure of Figure 2

\begin{tabular}{|c|c|c|c|c|c|c|c|}
\hline \multirow[b]{2}{*}{ Grid } & \multirow[b]{2}{*}{$N E$} & \multicolumn{3}{|c|}{ single loading case } & \multicolumn{3}{|c|}{ alternate loading case } \\
\hline & & It & $\mathrm{CPU}(\mathrm{s})$ & $W$ & It & $\mathrm{CPU}(\mathrm{s})$ & $W$ \\
\hline $13 \times 13$ & 14196 & 26 & 1.6 & 9.76232 & 23 & 8.6 & 2.49329 \\
\hline $25 \times 25$ & 195000 & 34 & 42.7 & 9.41125 & 40 & 335.0 & 2.42800 \\
\hline
\end{tabular}

Table 4 Results and statistics for the single loading using the procedure in Table 2 (structure of Figure 2)

\begin{tabular}{|c|c|c|c|c|c|c|c|c|c|c|c|c|}
\hline \multirow[b]{2}{*}{ Stage } & \multicolumn{4}{|c|}{$13 \times 13$ grid } & \multicolumn{4}{|c|}{$25 \times 25$ grid } & \multicolumn{4}{|c|}{$37 \times 37$ grid } \\
\hline & $N E$ & It & $\mathrm{CPU}(\mathrm{s})$ & $W$ & $N E$ & It & $\mathrm{CPU}(\mathrm{s})$ & $W$ & $N E$ & It & $\mathrm{CPU}(\mathrm{s})$ & $W$ \\
\hline 1 & 600 & 17 & 0.1 & 12.2500 & 2352 & 17 & 0.3 & 12.2500 & 5256 & 19 & 0.7 & 12.2500 \\
\hline 2 & 666 & 15 & 0.1 & 10.5625 & 2638 & 17 & 0.3 & 10.4011 & 5904 & 23 & 0.9 & 10.3103 \\
\hline 4 & 711 & 18 & 0.1 & 9.76559 & 3072 & 20 & 0.4 & 9.41410 & 7232 & 24 & 1.3 & 9.36663 \\
\hline 5 & 717 & 16 & 0.1 & 9.76232 & 3080 & 19 & 0.4 & 9.41134 & 7294 & 22 & 1.2 & 9.35113 \\
\hline 6 & 719 & 18 & 0.1 & 9.76232 & & & & & 7310 & 22 & 1.2 & 9.35109 \\
\hline
\end{tabular}

Table 5 Results and statistics for the alternate loading using the procedure in Table 2 (structure of Figure 2)

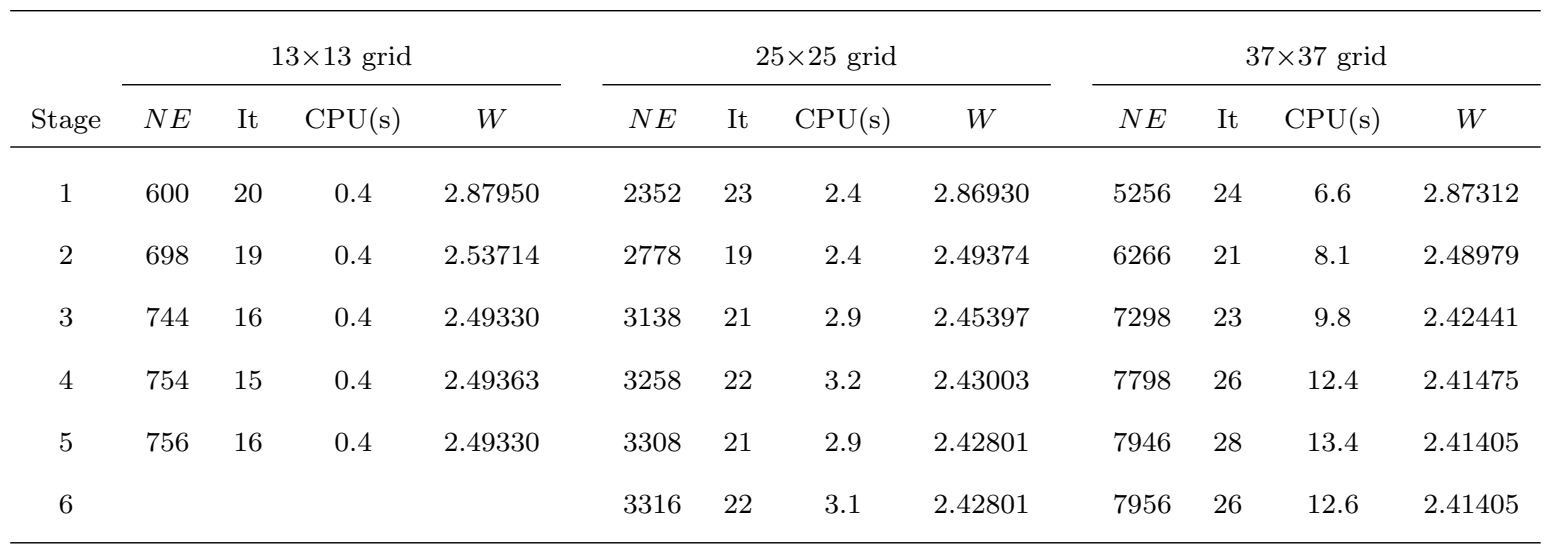


Table 6 Results and statistics for the $49 \times 97$ grid using the procedure in Table 2 (structure of Figure 2 )

\begin{tabular}{|c|c|c|c|c|c|c|c|c|}
\hline \multirow[b]{2}{*}{ Stage } & \multicolumn{4}{|c|}{ single loading case } & \multicolumn{4}{|c|}{ alternate loading case } \\
\hline & $N E$ & It & $\mathrm{CPU}(\mathrm{s})$ & $W$ & $N E$ & It & $\mathrm{CPU}(\mathrm{s})$ & $W$ \\
\hline 1 & 18576 & 20 & 3.3 & 12.2500 & 18576 & 30 & 40 & 2.86929 \\
\hline 2 & 22291 & 30 & 6.0 & 10.3765 & 23384 & 26 & 65 & 2.52488 \\
\hline 3 & 26749 & 35 & 9.2 & 9.61434 & 28060 & 31 & 94 & 2.46323 \\
\hline 4 & 32098 & 39 & 14 & 9.28835 & 33672 & 34 & 164 & 2.42347 \\
\hline 5 & 37820 & 37 & 17 & 9.23256 & 40406 & 36 & 247 & 2.40460 \\
\hline 6 & 38506 & 37 & 18 & 9.23078 & 48487 & 37 & 376 & 2.40175 \\
\hline 7 & 38834 & 37 & 18 & 9.23058 & 51392 & 37 & 426 & 2.40144 \\
\hline 8 & 38867 & 37 & 17 & 9.23053 & 51734 & 35 & 402 & 2.40142 \\
\hline 9 & 38870 & 37 & 17 & 9.23061 & 51786 & 35 & 343 & 2.40142 \\
\hline 10 & & & & & 51792 & 36 & 384 & 2.40142 \\
\hline
\end{tabular}

Table 7 Results and statistics for the cantilever beam using the procedure in Table 2. The compliance has been multiplied by $E V / P^{2} L^{2}$ and is given in the $W$ column

\begin{tabular}{|c|c|c|c|c|c|c|c|c|c|c|c|c|}
\hline \multirow[b]{2}{*}{ Stage } & \multicolumn{4}{|c|}{ case III } & \multicolumn{4}{|c|}{ case IV } & \multicolumn{4}{|c|}{ case $\mathrm{V}$} \\
\hline & $N E$ & It & $\mathrm{CPU}(\mathrm{s})$ & $W$ & $N E$ & It & $\mathrm{CPU}(\mathrm{s})$ & $W$ & $N E$ & It & $\mathrm{CPU}(\mathrm{s})$ & $W$ \\
\hline 1 & 3260 & 20 & 1.5 & 16.4434 & 3260 & 17 & 0.5 & 9.00000 & 3260 & 30 & 7.1 & 9.20314 \\
\hline 2 & 3912 & 32 & 2.9 & 15.1689 & 3850 & 23 & 0.6 & 8.26874 & 3899 & 28 & 10.9 & 8.52115 \\
\hline 3 & 4694 & 32 & 3.8 & 14.9443 & 4620 & 29 & 0.9 & 8.09550 & 4678 & 28 & 14.6 & 8.33287 \\
\hline 4 & 5632 & 31 & 5.1 & 14.6396 & 5544 & 30 & 1.1 & 7.87394 & 5613 & 31 & 19.7 & 8.19506 \\
\hline 5 & 6758 & 33 & 7.1 & 14.4778 & 6652 & 38 & 1.7 & 7.80953 & 6735 & 35 & 29.1 & 8.02766 \\
\hline 6 & 8013 & 36 & 9.2 & 14.4606 & 7178 & 33 & 1.7 & 7.80223 & 8082 & 37 & 38.4 & 7.98871 \\
\hline 7 & 8194 & 38 & 10.5 & 14.4604 & 7284 & 31 & 1.6 & 7.80172 & 9107 & 40 & 51.4 & 7.98542 \\
\hline 8 & 8221 & 36 & 9.7 & 14.4604 & 7297 & 32 & 1.6 & 7.80172 & 9230 & 36 & 48.9 & 7.98537 \\
\hline 9 & 8228 & 35 & 9.7 & 14.4604 & 7298 & 32 & 1.6 & 7.80172 & 9291 & 35 & 43.1 & 7.98537 \\
\hline 10 & & & & & & & & & 9296 & 36 & 45.5 & 7.98537 \\
\hline
\end{tabular}

Table 8 Results and statistics for structures A,B, and C of Figure 9

\begin{tabular}{|c|c|c|c|c|c|c|c|c|c|c|}
\hline \multirow[b]{2}{*}{ MESH } & \multirow[b]{2}{*}{$N E$} & \multicolumn{3}{|c|}{ Structure A } & \multicolumn{3}{|c|}{ Structure B } & \multicolumn{3}{|c|}{ Structure C } \\
\hline & & It & $\mathrm{CPU}(\mathrm{s})$ & $W$ & It & $\mathrm{CPU}(\mathrm{s})$ & $W$ & It & $\mathrm{CPU}(\mathrm{s})$ & $W$ \\
\hline $20 \times 40$ & 3200 & 31 & 8.7 & 11.630 & 31 & 8.7 & 10.748 & 28 & 7.6 & 1.4853 \\
\hline $40 \times 80$ & 12800 & 38 & 48.7 & 11.640 & 36 & 47.0 & 10.756 & 32 & 41.5 & 1.4892 \\
\hline
\end{tabular}




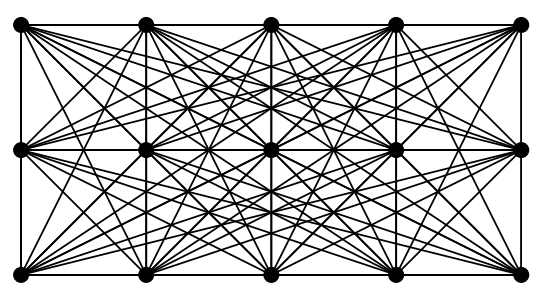

(a)

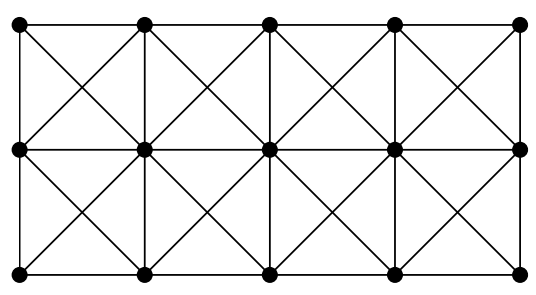

(b)

Fig. 1 Grid of $3 \times 5$ nodes (a) fully connected ground structure (b) partially connected

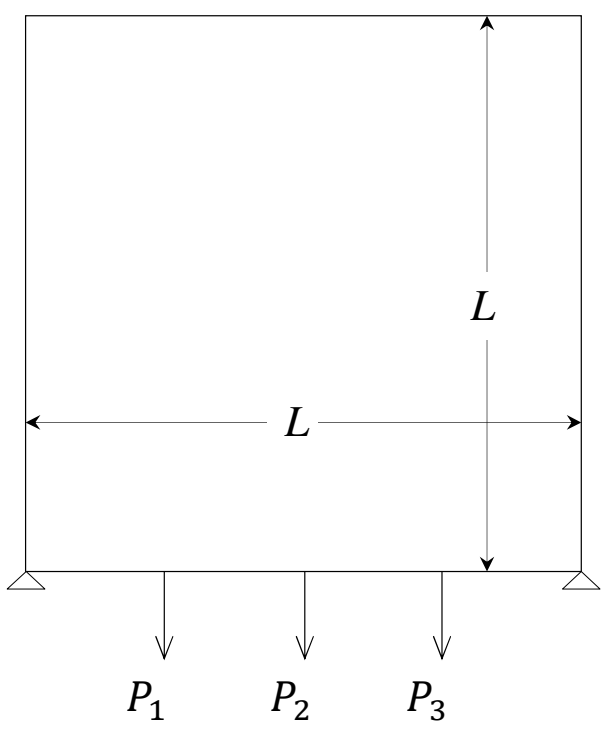

Fig. 2 Example 1: Box shaped structure subjected to point loads 


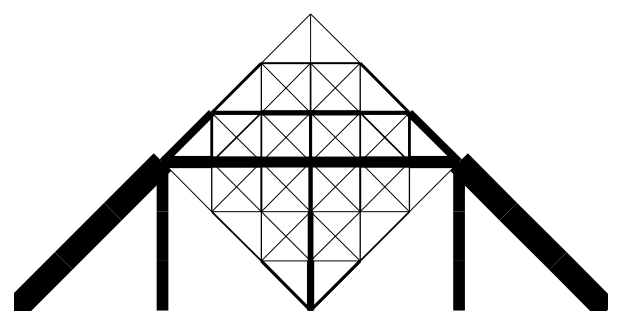

stage 1

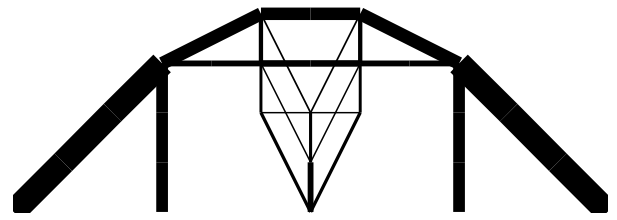

stage 2

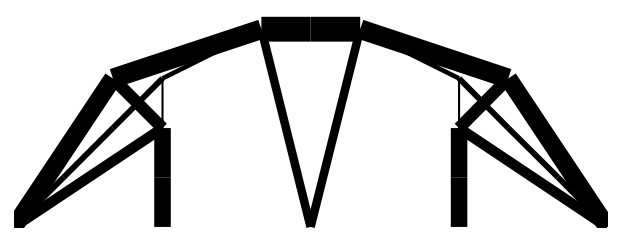

stage 3

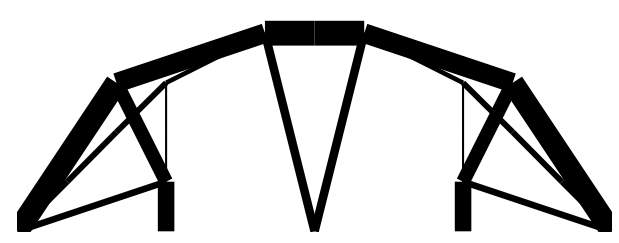

stage 4

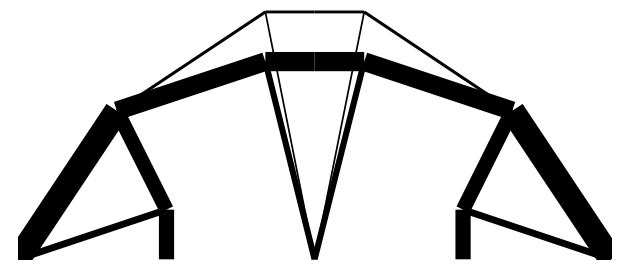

stage 5,6

(a)

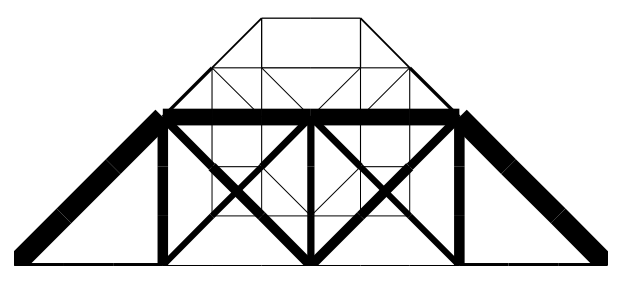

stage 1

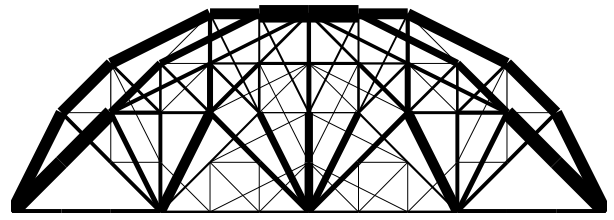

stage 2

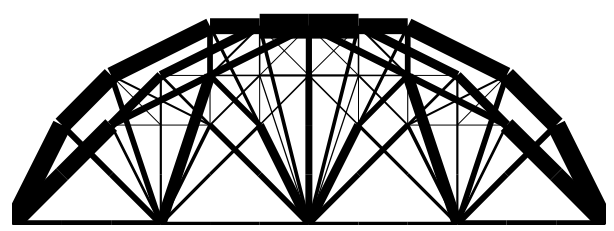

stage 3

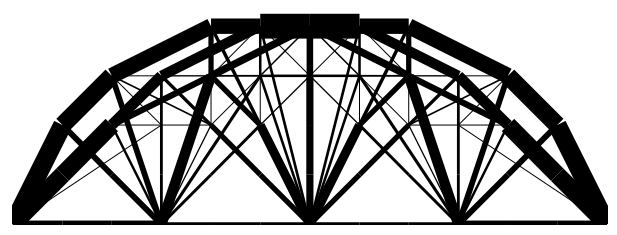

stage 4

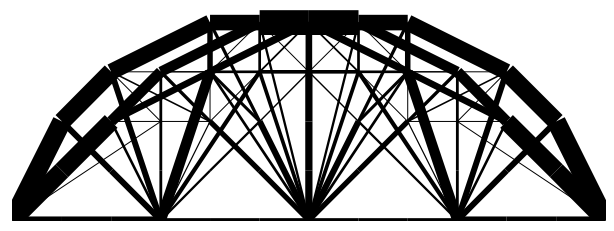

stage 5

(b)

Fig. 3 Example 1: Optimal layout for the $13 \times 13$ grid (a) single loading - layout for the stages 1-5 (stage 6 is identical) (b) alternate loading - layout for all the stages 


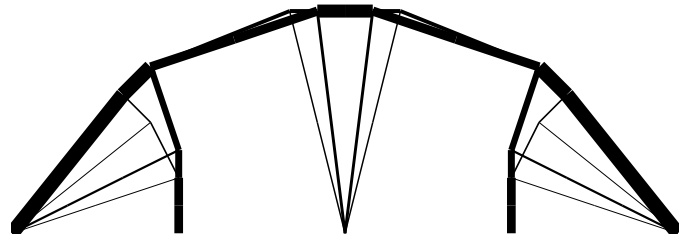

$25 \times 25$ grid

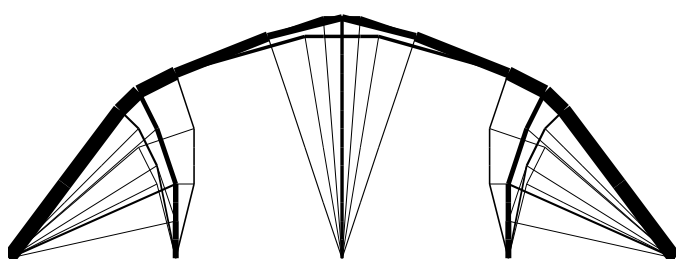

$37 \times 37$ grid

(a)

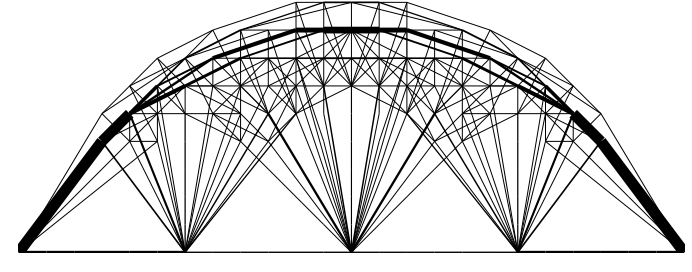

$25 \times 25$ grid

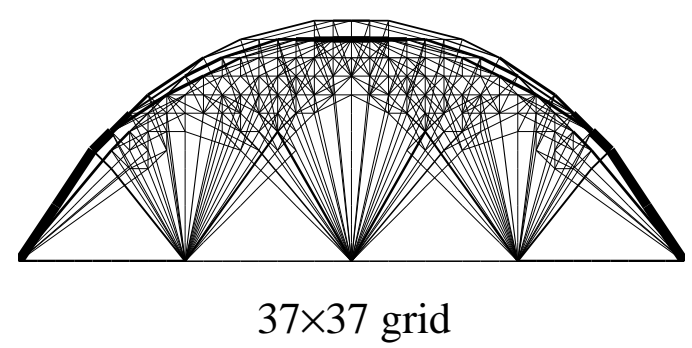

(b)

Fig. 4 Example 1: Final optimal layouts (a) single loading for the $25 \times 25$ and the $37 \times 37$ grid (b) alternate loading for the $25 \times 25$ and the $37 \times 37$ grid 


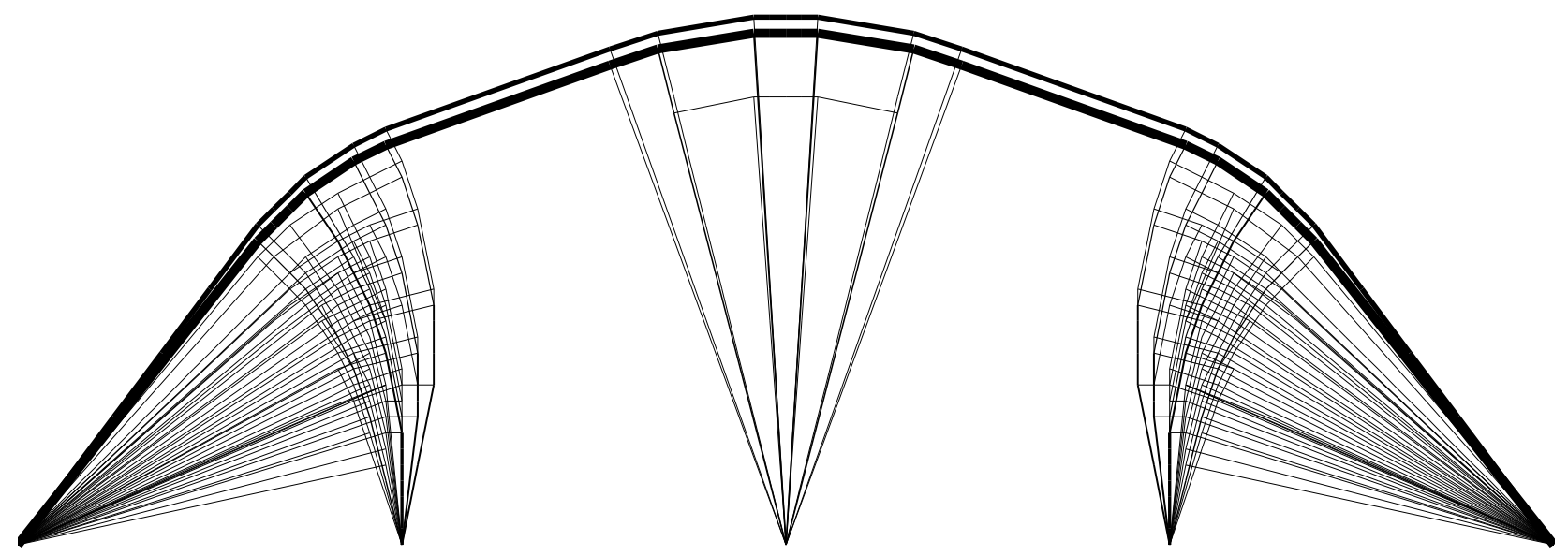

Fig. 5 Example 1: Final optimal layout for single loading for the $49 \times 97$ grid

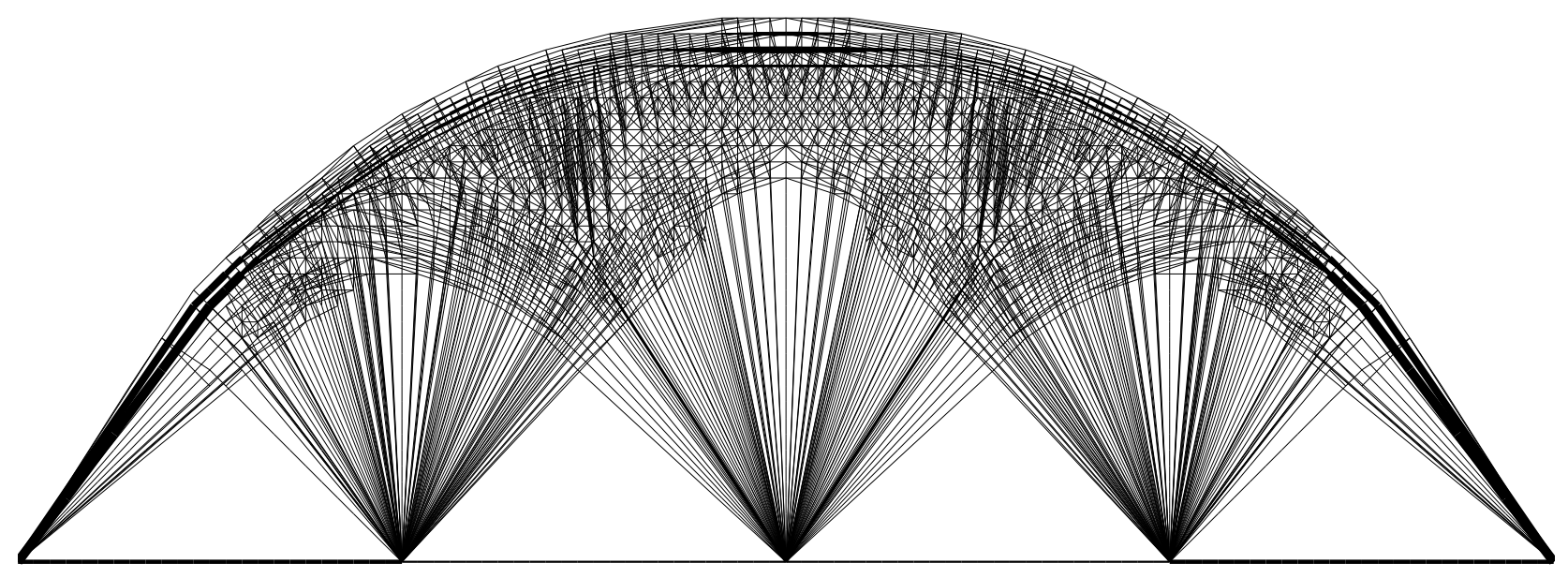

Fig. 6 Example 1: Final optimal layout for alternate loading for the $49 \times 97$ grid 


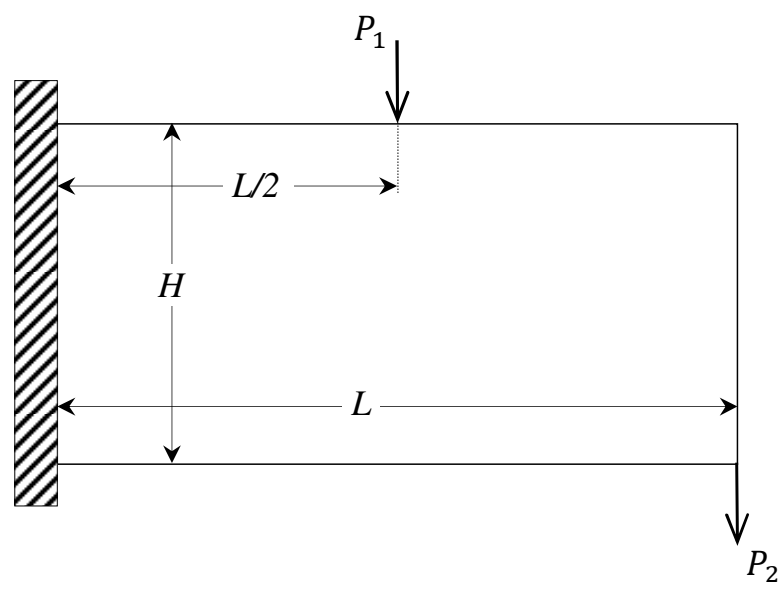

Fig. 7 Notation for the cantilever beam

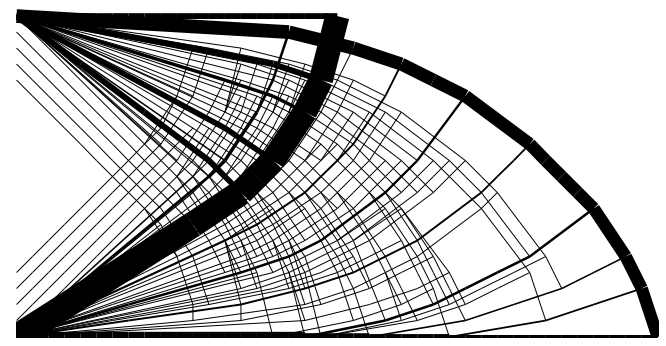

(a)

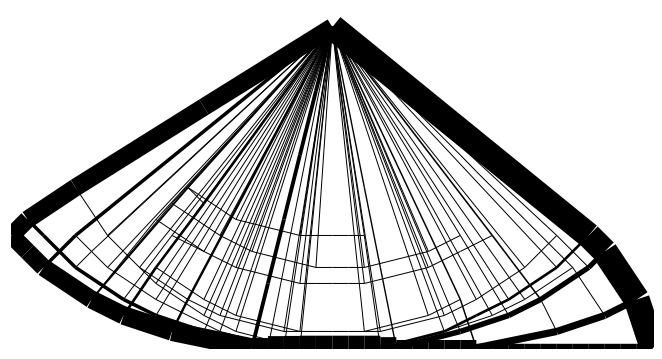

(c)

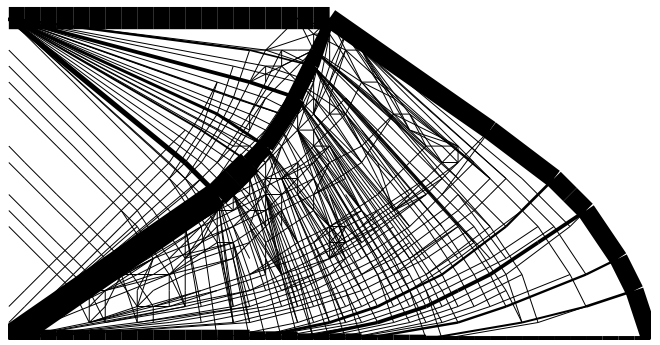

(b)

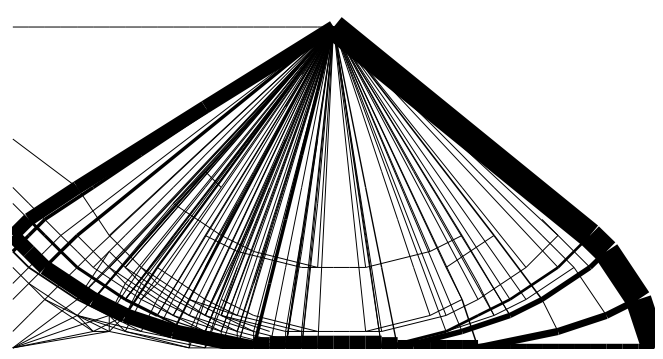

(d)

Fig. 8 Optimal layouts for the clamped beam of Figure 7 (a) Case I, $N E=7146$, and $W=40.481$ (Case II is similar but with 7087 members), (b) Case III, $N E=8228, W=14.460$ (c) Case IV, $N E=7298, W=7.802$, (d) Case V, $N E=9296,, W=7.985 . W$ is the compliance multiplied by $E V / P L^{2}$ 


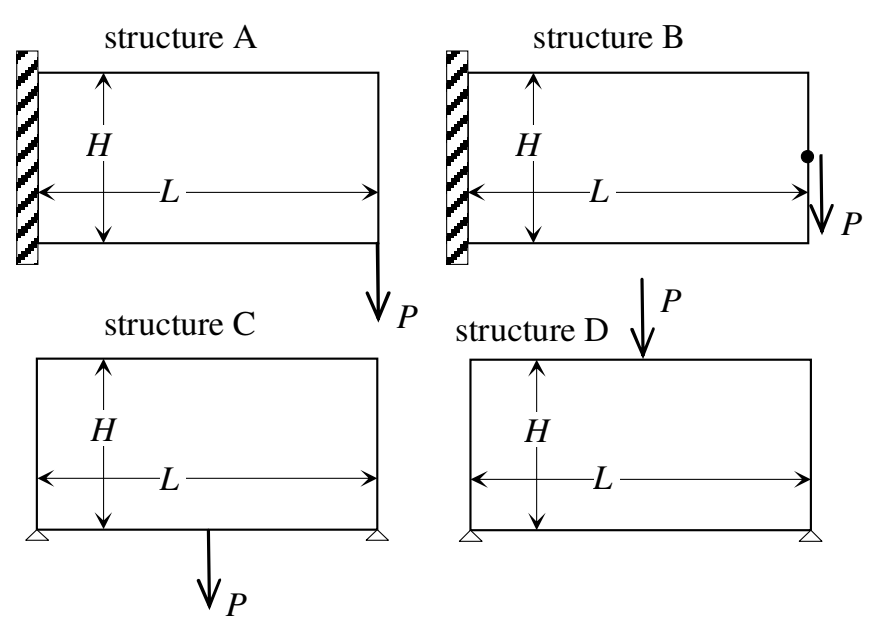

Fig. 9 Notation of various structures 
structure A, mesh $20 \times 40$ thickness contour

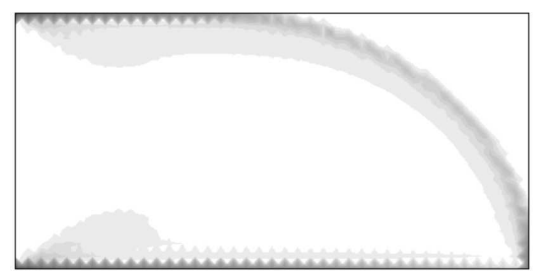

structure A, mesh $20 \times 40$ scaled thickness contour

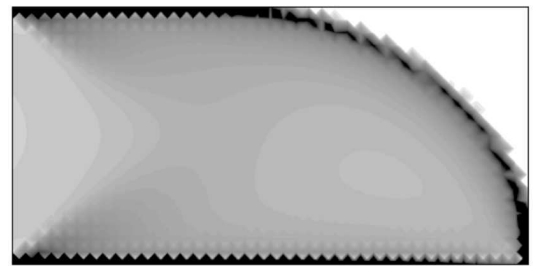

structure B, mesh $40 \times 80$ scaled thickness contour
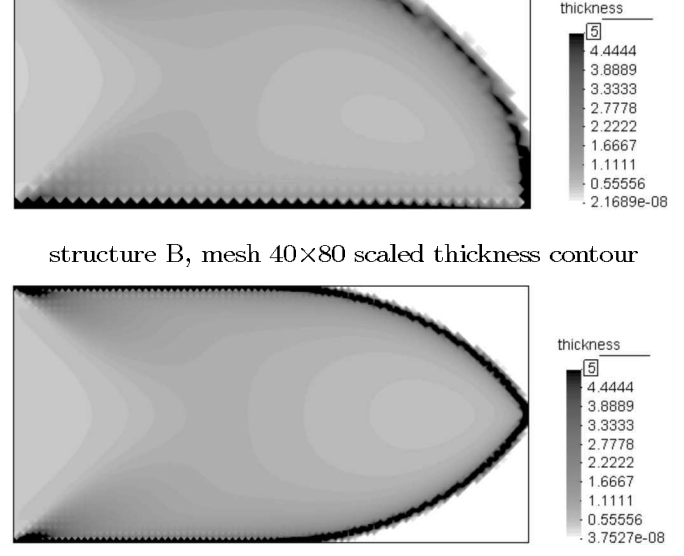

structure D, mesh $20 \times 40$ thickness contour

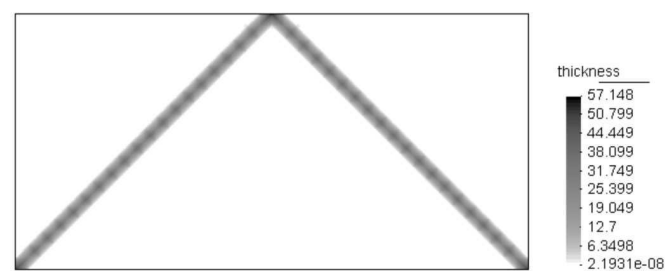

structure A, mesh $40 \times 80$ thickness contour

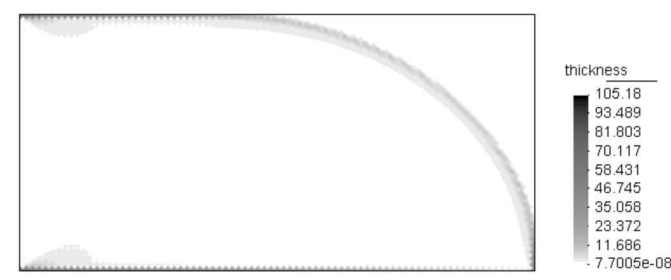

structure A, mesh $40 \times 80$ scaled thickness contour

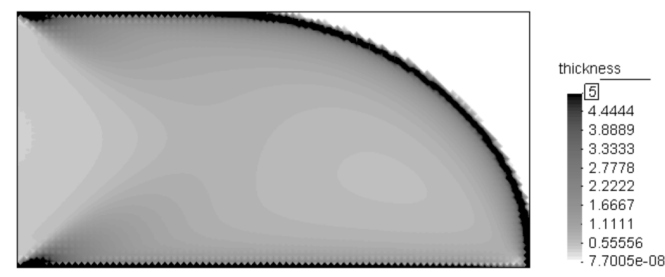

structure C, mesh $40 \times 80$ scaled thickness contour

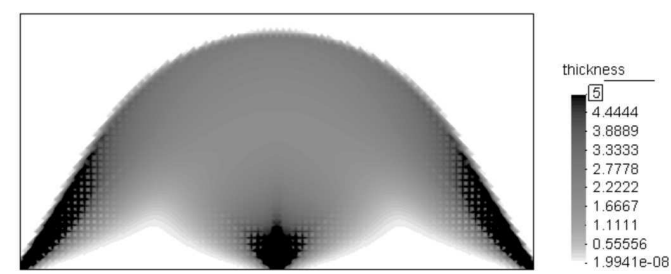

Fig. 10 Optimal contour thickness for various cases for the structures of Figure 9 


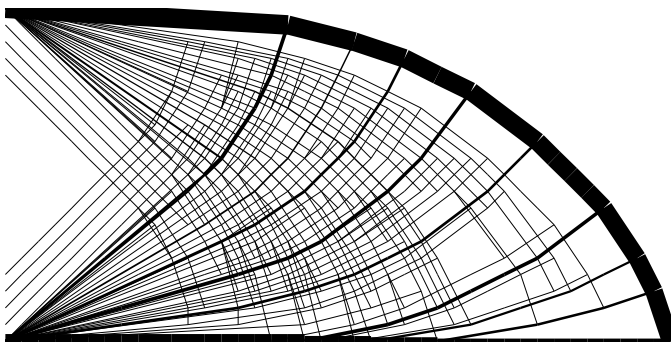

(a)

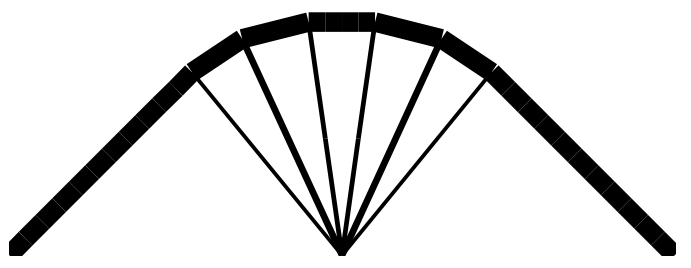

(c)

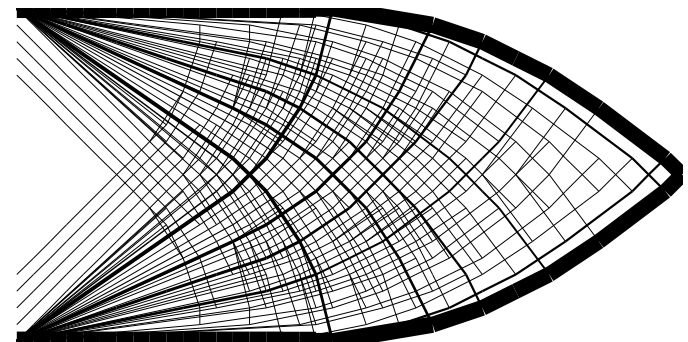

(b)

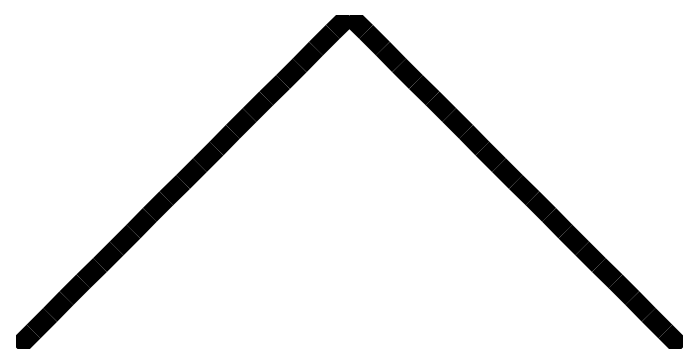

(d)

Fig. 11 Optimal layouts for the structures of the Figure 9 considering truss discretizations and $21 \times 41$ grid (a) structure A, 7011 members, $W=13.335$ (b) structure $\mathrm{B}, 7077$ members, $W=12.409$ (c) structure $\mathrm{C}, 4882$ members, $W=1.6663$ (d) structure $\mathrm{D}, 4108$ members, $W=1.0$ 